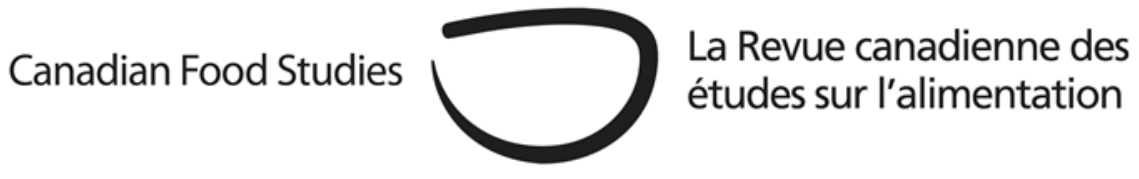

Original Research Article

\title{
A participatory study of the health and social impact of a food centre in Ottawa, Canada
}

\author{
Aganeta Enns ${ }^{a^{*}}$, Myddryn Ellis ${ }^{b}$, Tracey O'Sullivan ${ }^{a}$, Peter Milley ${ }^{a}$, and Elizabeth Kristjansson ${ }^{a}$

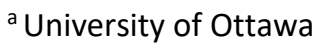 \\ ${ }^{\mathrm{b}}$ McGill University
}

\begin{abstract}
Food insecurity is a pervasive and persistent issue across Canada, where a growing number of people are accessing food banks. Conventional food banks may offer relief for immediate needs but typically have limited capacity to address longer-term food insecurity. This paper focuses on the Parkdale Food Centre in Ottawa, ON, which provides food assistance alongside a range of programs and initiatives designed to address food insecurity and related needs in its community. This qualitative study aims to examine how participation in the programs at the Parkdale Food Centre influences the physical, mental, and social health of people who access the food centre. Semi-structured interviews and a participatory photovoice project were conducted with people who access the food centre. The results indicate that people who access the food centre perceived a positive influence of the programs on their food, health, and social needs, particularly with respect to improved access to fresh foods, connections to social and health resources, and reduced stigma. Incorporating additional food, health, and social programming into an existing food assistance program may help ameliorate elements of food insecurity and associated negative outcomes within communities impacted by this issue.
\end{abstract}

Keywords: Food insecurity; photovoice; qualitative research; food banks; food centres Introduction

*Corresponding author: aenns022@uottawa.ca 
Food insecurity, which refers to having inadequate or uncertain access to a sufficient quantity of food and/or inadequate quality and variety of foods due to financial constraints, is a persistent issue in communities across Canada that has a negative impact on approximately $8.3 \%$ of households (Roshanafshar \& Hawkins, 2015). Experiences of food insecurity can range from worrying about food running out to skipping meals or going without eating for a whole day (Tarasuk et al., 2016).

Food insecurity has been linked with consuming a lower quality diet and with poorer nutrient intake (Kirkpatrick \& Tarasuk, 2008). Compared to food secure populations, people experiencing food insecurity report consumption of fewer fruits and vegetables and regular intake of more foods that are inexpensive, calorically dense, and low in nutrients (Holben, 2012; Neter et al., 2017). These calorically dense but low-nutrient foods may help connect the paradoxical finding that food insecure populations, particularly women, are at an elevated risk of both lower nutrient intake and obesity (Cheug et al., 2015; Laraia, 2013). Prior studies indicate that food insecurity is associated with increased risk of poorer overall physical health and chronic disease (Ramsey et al., 2011; Seligman et al., 2010). Furthermore, adverse mental health outcomes have been linked with food insecurity, including higher levels of perceived stress, symptoms of depression and anxiety, and lower self-esteem (Laraia, 2013; Leung et al., 2015; Pryor et al., 2016). People impacted by food insecurity also tend to report lower social support and community cohesion (Boston et al., 2013; Gichunge et al., 2015; Leung et al., 2015).

Interventions to address food insecurity

In Canada, food banks were initially established in the 1980s as a resource for short-term relief of urgent food need. They are typically one of the only sources of immediate assistance for food insecure individuals. While the organizational structures of these agencies differ, they often rely on charitable donations from public and private sources (Campbell et al., 2013; Tarasuk et al., 2014). In the context of the present study, food banks are defined as not-for-profit agencies that distribute food, typically in the form of grocery items, to individuals. Food banks are often limited in their capacity to provide adequate quantities of food to meet clients' needs and frequently need to implement access restrictions to ensure their food supply can meet the demand (Tarasuk et al., 2014). The limited variety and quality of foods available, including few fresh and nutritious options, has been described in previous literature (e.g., Gany et al., 2013; Tarasuk et al., 2014). In addition to food supply limitations, clients may experience social stigma or shame when accessing a food bank (Van der Horst et al., 2014). People who are food insecure may view the embarrassment or shame of seeking assistance at a food bank as a barrier to accessing these resources. However, people who access food banks have reported a sense of relief if they have a welcoming experience when accessing the food bank (Purdam et al., 2015).

In one month in 2016, Food Banks Canada reported that 860,000 people across the country accessed a food bank, which represents a $28 \%$ increase compared to 2008 (Food Banks 
Canada, 2016). While food banks can facilitate short-term improvement of food security (Roncarolo et al., 2016), they often have a limited capacity to help clients meet their dietary needs and alleviate chronic food insecurity. Despite this, many families rely on these resources to meet their monthly household food needs (Feeding America, 2014). With the increasing number of people turning to food banks, and many relying on these resources on a long-term basis, some observers have noted that conventional food banks have limited capacity to address the causes of food insecurity (Loopstra \& Tarasuk, 2012; McIntyre et al., 2016). It has been argued that the ubiquity of food banks may even diminish public perceptions that people are entitled to adequate access to food and that formal government responses to this issue are needed (Riches \& Silvasti, 2014). Traditional food banks were not intended to address long-term food insecurity or the causes of this issue (Riches \& Silvasti, 2014), but these established community agencies may offer an effective setting for advocacy and for targeting resources to address factors that contribute to food security (Greater Vancouver Food Bank, 2018).

\section{Shifting strategies in food banking}

A recent review suggests that food security outcomes may improve when food banks work to identify and address the food needs of the people they serve and offer more perishable foods (Bazerghi et al., 2016). Descriptions of how food banks have adapted existing operations or adopted new programs to better address the needs of the people they serve have emerged in recent literature. For example, a survey of food banks in the United States found that nutritional policies and efforts to increase the availability of fresh produce are increasingly common (Campbell et al., 2013). Food banks have changed how they operate and have adopted new programs in order to offer more nutritious foods (including produce), nutrition education, cooking classes, and food demonstrations, resulting in reported improvements in dietary outcomes (Caspi et al., 2016; Dave et al., 2017; Handforth et al., 2013; Martin et al., 2013).

There has been heterogeneity in the cases of food banks that have shifted how they operate to better meet a wide range of needs of the people who access food banks. For example, it has been reported that the dignity of clients is promoted when food banks allow individuals to choose the food items they wish to access rather than providing food hampers, which are orders (e.g., bags or boxes) of grocery items that are pre-packed by volunteers or staff for distribution to individuals who access the food banks (Mukoya et al., 2017). Programs to empower and support individuals in developing skills, finding employment, budgeting, and building self-efficacy have also been implemented successfully at food banks. Food banks may be an effective community access point for offering targeted food security, health, advocacy, and social programs (Greater Vancouver Food Bank, 2016; Levkoe \& Wakefield, 2011).

The Parkdale Food Centre (PFC) in Ottawa, ON is an example of a centre that began as a food bank but radically changed its approach to addressing food insecurity by drawing on a number of the strategies discussed above and inventing their own. Their approach is described in 
more detail below. The purpose of the present study is to conduct a pilot study with the Parkdale Food Centre to examine the influence of their approach on the community members it is intended to serve. In this paper, we describe participants' experiences with PFC, including the social and emotional experiences of accessing a food centre, and how they perceive changes in their diet, physical health, mental health, and social support since engaging with PFC.

\section{Methodology}

\section{Study context}

The study was conducted at the Parkdale Food Centre, which is located in an urban area of Ottawa, ON. This not-for-profit organization is part of the Ottawa Food Bank's distribution network. The mission statement at PFC is "to build healthier, more connected neighbours and neighbourhoods through good food, innovative community partnerships, and by challenging inequalities in order to create lasting impacts" (Parkdale Food Centre, 2019). A distinguishing feature of PFC is that they refer to people who access the centre as neighbours to emphasize a welcoming sense of community. PFC provides food assistance with a focus on providing nutritious foods, including fresh produce, and avoiding low-nutrient 'junk' foods. They encourage donors to give healthy foods and provide a "good foods list" for suggested donation items. Rather than distributing food hampers, PFC employs a "choice model" to distribute orders of grocery items to those seeking food assistance. People who access PFC are invited to walk around a food display area with a volunteer and select the foods within each category that they would like to take home. Approximately one-third of the people who access PFC reside outside of the centre's defined region in Ottawa. People seeking assistance from outside the defined region were referred by healthcare professionals to PFC so they can obtain access to more fresh and healthier foods. Individuals may access PFC for an order of grocery items once per month; however, there are no restrictions on how often individuals may access PFC for other services or programs.

PFC focuses on social justice, community awareness, and advocacy efforts for changing policies to better address issues of food insecurity. PFC facilitates community programs onsite at the Food Centre that are available to all people who access PFC. Examples of programs include: cooking classes, community meals, nutrition information programs, a community garden, financial advice meetings, programs facilitated by a social worker, and referrals to a broad range of other community resources. Many of the neighbours volunteer with the various programs at the centre. While PFC still offers food banks services (i.e., distributing food directly to individuals), it is herein referred to as a food centre rather than food bank to better represent the 
scope of food and community programs offered in addition to food bank services as they have been defined above.

\section{Study context}

The overarching research questions for this study were:

1. How do participants perceive their experiences, including social and emotional experiences, in accessing programs and services at PFC?

2. How do participants describe the effect of accessing PFC on their lives, including aspects of their diet, health, and social experiences?

A multiple-methods qualitative approach was employed to investigate the study aims. The study was conducted over a one-year period that involved a participatory photovoice project and semi-structured interviews that were conducted before the photovoice project at the beginning of the study period and a follow-up cycle of semi-structured interviews that were conducted after the photovoice project at the end of the study period.

\section{Data collection}

The sample of 12 participants took part in initial semi-structured interviews at the beginning of the study period conducted in a private room located in the same building as PFC. The interviews ranged in duration from 20 to 55 minutes. The interview guide included questions pertaining to overall experience and interactions at PFC, as well as perceived diet, physical health, mental health, and social support. Data were transcribed verbatim and anonymized prior to analysis.

After completing the initial interviews, the 12 participants were then invited to take part in the photovoice project. Photovoice (Wang \& Burris, 1997) was selected to incorporate an established participatory methodology that would allow participants to photograph and narrate their experiences in interacting with the Food Centre. Photovoice was selected in combination with interviews to empower participants to identify topics, concerns, and experiences that are most salient from their perspectives (Palibroda et al., 2009). This method allows participants to present priority concerns, create a critical dialogue, share experiences, and reflect on strengths through photographs and discussions. Moreover, previous literature has indicated that photovoice expands the representation of participant voices and realities that are not often represented in research (Hergenrather et al., 2009). Incorporating a visual method (i.e., sharing photographs in the photovoice project) was used as a means for participants to share what is most prominent and important from their perspectives and stimulate discussion about experiences at PFC (HansenKetchum \& Myrick, 2008; Wang \& Burris, 1997). The photovoice project took place over the course of five group meetings, held at approximately one-month intervals. The average duration of the group meetings was approximately one hour. At the first meeting, participants were 
provided with information on photovoice, shared photography tips, and learned how to use the cameras provided. This meeting was for information purposes and was not audio-recorded. Participants were asked to take photos that show their experiences as they relate to programs at PFC. At the second to fifth group meetings, participants shared their photos and discussed the meaning and relevance of each photo. The photos stimulated open group discussions on experiences of accessing PFC where photographs guided the dialogue and critical group discussions and allowed participants to identify the most important and salient topics. These discussions were audio-recorded and transcribed verbatim.

After the photovoice project concluded, all 12 participants that completed the initial interviews were invited to complete a follow-up interview at the end of the study period. They were asked about their experiences and interactions at PFC since their initial interviews, including if and how the programs at PFC have impacted their lives. They were prompted to provide any feedback on aspects of PFC that they believe could be improved. Data collection occurred between March 2016 and March 2017. Approval was obtained from the University of Ottawa Research Ethics Board prior to data collection.

\section{Participants}

Twelve adults who access PFC were recruited to participate. Participants were recruited inperson at PFC. Two research team members approached people in the waiting area of PFC to provide information on the study and invite individuals to participate. People who expressed interest in participating were asked to write their preferred contact information (e.g., phone or email) and place it in a locked drop box. The research team then contacted participants to schedule one-on-one interviews. The sample was limited to participants who were proficient in English and were over the age of 18 . The sample included six female and six male participants whose ages ranged from 29 to 61 . Four of the 12 participants reported living with dependent children and two indicated current pregnancy. Length of time since first accessing the services and programs at PFC ranged from six months to over five years. All participants reported accessing other food banks prior to seeking assistance at PFC. Two of the 12 participants indicated they were employed at the time of the initial interview.

Four of the initial 12 participants interviewed completed the photovoice project. All 12 participants who completed the initial interview were contacted with an invitation to complete a follow-up interview at the end of the study period. Five of the 12 participants, including all four participants who took part in the photovoice project, completed the one-year follow-up interviews. Three participants were lost to follow-up after completing the initial interview because the research team was unable to make contact (e.g., out-of-service phone number). The remaining participants who did not complete all aspects of data collection explained they were unable to commit their time to the study due to health or other issues of personal circumstance. This attrition is consistent with previous literature that has documented the challenges of 
retaining participants from groups that are hard-to-reach or may be considered marginalized (e.g., McKenzie et al., 1999). All participants reviewed and signed a consent form approved by the University of Ottawa ethics review board.

\section{Data analysis}

All one-on-one interviews and photovoice meetings two through five were audio-recorded and transcribed verbatim. Thematic analysis was conducted with the interview transcripts and the data collected through the photovoice group meetings to summarize emergent themes (Thomas, 2006). The data were coded and analyzed using NVivo10. Codes were developed by two independent coders and agreed on by the research team. Codes triangulated from the baseline interviews and the photovoice data were summarized and brought to the follow-up interviews with participants, who were asked to read the data analysis summary and confirm whether they perceived it to be accurate, what they would change or add, or any other thoughts they had on the summary. This feedback from participants was then used to refine the final themes. This last step also involved triangulating themes from both the photovoice and interview data, during which the research team analyzed coding reports to identify themes through iterative revisions until consensus was reached. Verbatim quotes from interviews and photovoice meetings, as well as select photographs that had been shared in the photovoice project, are provided below to illustrate these themes. Photographs shown were limited to those that do not contain identifying details in order to protect participants' confidentiality.

\section{Results}

Participant experiences at PFC and the perceived influence of accessing PFC have been summarized into three themes based on the triangulated analyses of the interview and photovoice data: food and nutrition, the community hub, and dignity. The three themes summarize how participants described the influence of PFC on their access to food, including healthy food options, connections within the community, and overall social and emotional experiences of accessing the Food Centre.

\section{Food and nutrition}

Participants described the food offered at PFC as being of suitable quantity, quality, and variety to meet their food needs. Participants discussed the wide variety of foods, the availability of fresh fruits and vegetables, and described being satisfied with the quantity of food received. A photograph shared by one participant of the photovoice project, shown in Figure 1, shows a display of fresh produce at PFC. 
Figure 1: Photograph of fruits and vegetables taken at PFC.

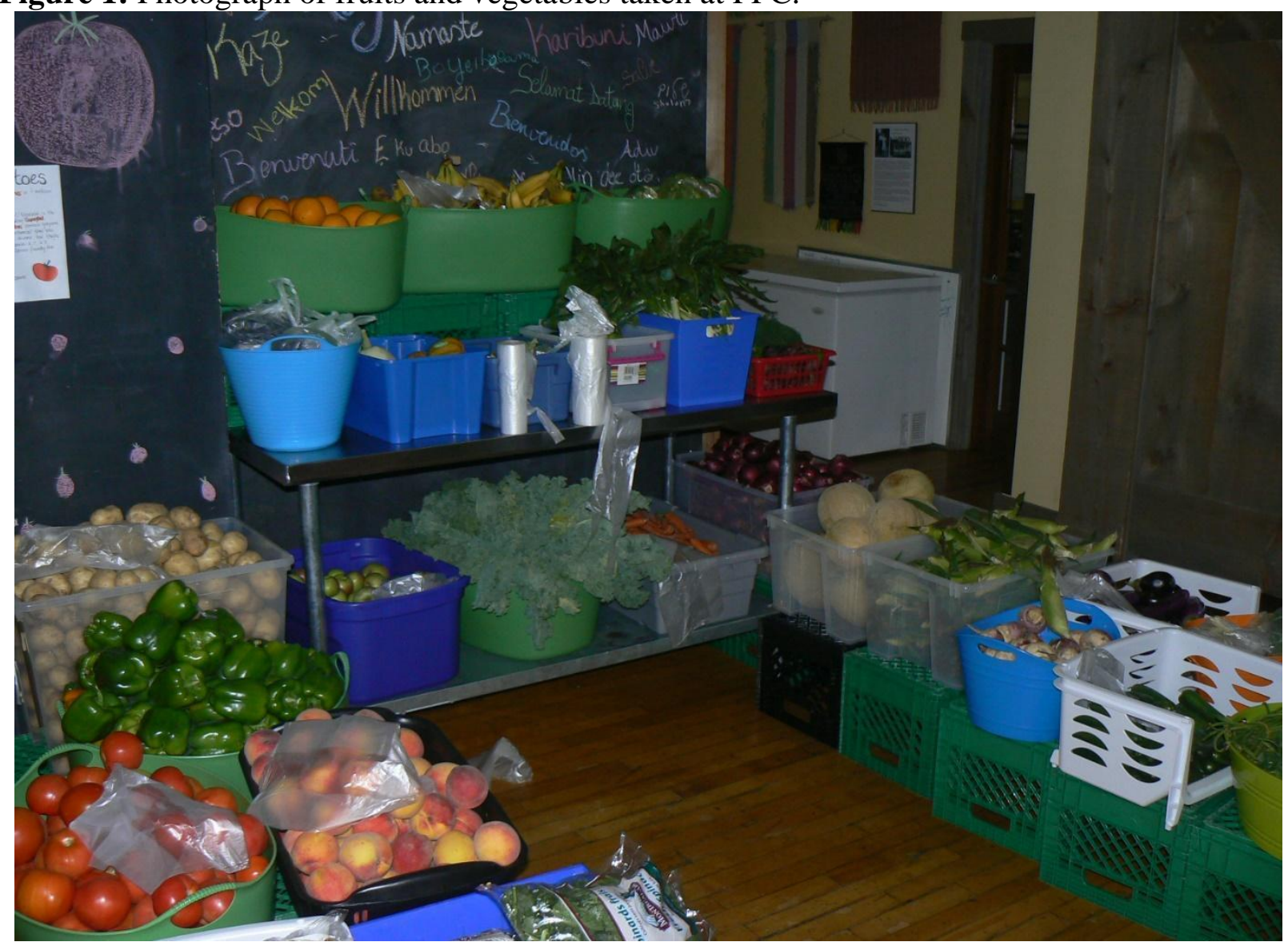

In the initial interviews, nine out of the 12 participants interviewed mentioned the high quality, freshness, and/or variety of foods available at PFC. The participant who shared the photograph shown in Figure 2 explained that these were grocery items obtained from the Food Centre and discussed the amount and variety of fruits and vegetables that they obtained from PFC. During the interviews and photovoice project, participants also spoke favourably about their access to food at PFC in comparison to food they had received at other food banks or with respect to their difficulties in purchasing food for a healthy diet.

It's the healthiest food bank I've ever been to around here. Actually, because I find a lot of food banks are all high carbs, high carbs to fill you up or whatever. This is, [PFC] look for your health. They give you good foods to eat, and it's the proper foods to eat. It's good. (Participant 6) 
Figure 2: Photograph of grocery items obtained at PFC.

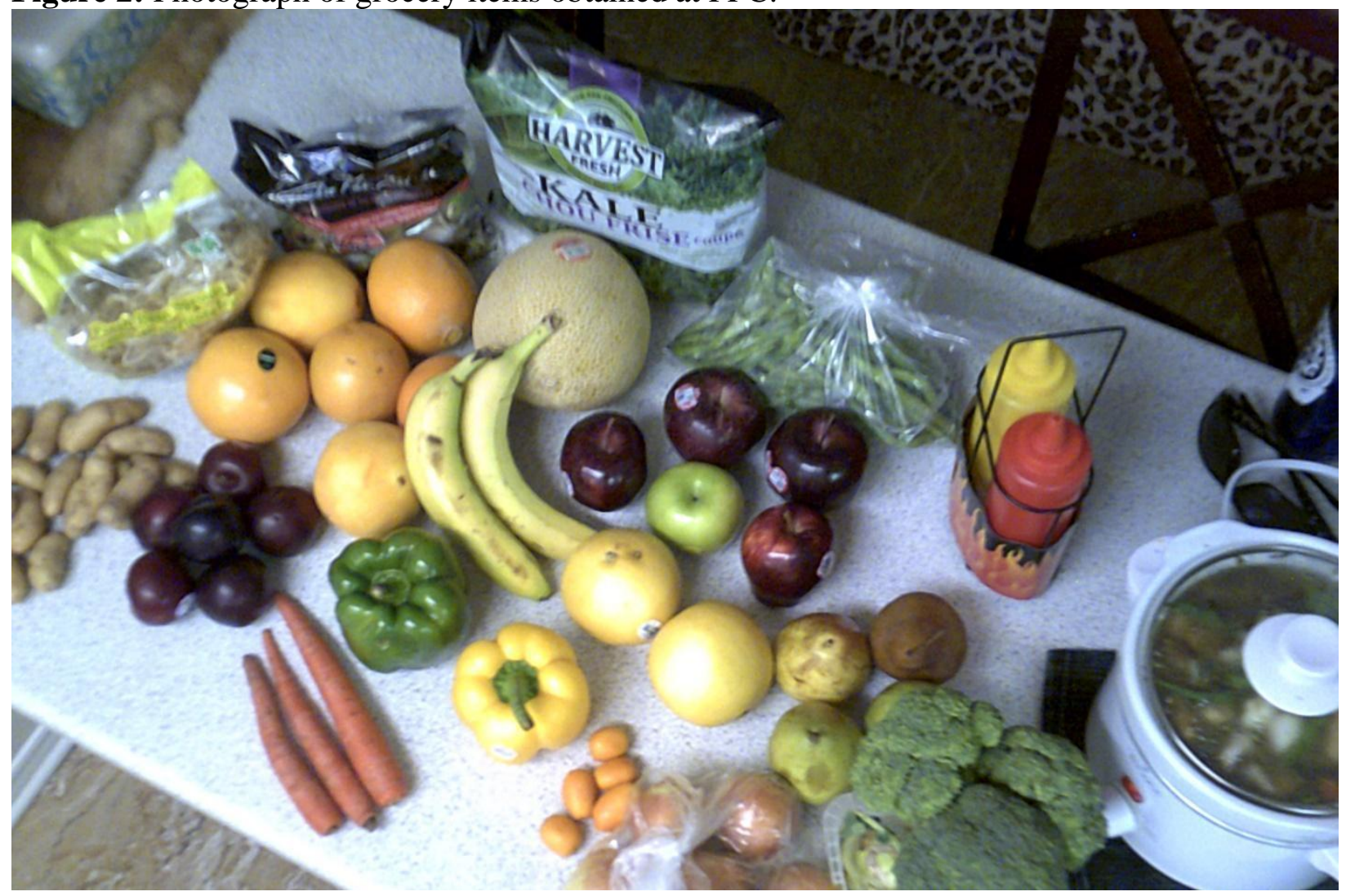

Several participants associated their access to services and programs at PFC with eating a healthier diet. Examples they provided about their healthier eating included reduced consumption of 'junk' food, increased consumption (in quantity and variety) of fruits and vegetables, and more frequent preparation of 'healthy' meals. One participant discussed how these dietary changes helped them to meet a goal to reach a healthy body weight. Participants perceived food options as being accessible for those with specific dietary needs related to managing chronic conditions or other dietary restrictions.

They offer fresh fruits and vegetables and they also give you foods that you can make instead of pre-packaged. So, it's a lot healthier. And that helps you if you want to have a healthier diet. (Participant 11)

I'm eating more now. I used to eat once a day thinking I'm going to get fat. Now they're $[\mathrm{PFC}]$ teaching me about eating nutritionally and I'm eating more and I'm not getting fat, I'm losing weight. (Participant 10)

Information and programs on nutrition and cooking facilitated at PFC were discussed in eight interviews. Participants discussed learning new information and skills at the programs facilitated at PFC, including the regular community kitchen programs. Participants also described learning new information through the food of the month program at PFC, recipes, and 
information provided by volunteers or staff on how to prepare and incorporate new foods into meals.

At the food bank, they're starting to do these things now where they're showing you what sort of foods you can make with the actual fruit or the vegetable, how you can incorporate it, the calories, everything, because it's so important. (Participant 7)

The community hub

Several participants discussed the role of PFC in relation to their social support and sense of community belonging. They explained the need for space in the community to meet and talk with other people. PFC was described as a place to drop-in for social interaction with others.

Participants talked with their neighbours in the waiting area and explained that they felt more connected in their community because of interactions at PFC.

I just continued to come along for the socialization right, when you move to a city, besides your family, who are you going to socialize with? The centre's been helpful, just to be able to come here, a dependable place where the hours are dependable where you can count on it being open, there's coffee. (Participant 1)

To me this is very much a social service. The cooking classes and so forth because, well I can drop in here any day it's open. (Participant 12)

Involvement in programs facilitated at PFC, such as the community meals, emerged in several interviews and group discussions. Moreover, participants described the success PFC has had in connecting with local businesses and the positive impact of these community connections. The neighbours discussed the donations received by local businesses, the presence of PFC in the community, and events or programs offered jointly by PFC and local businesses. One participant shared a photograph taken at a breakfast event that was offered jointly by PFC and a local restaurant (Figure 3). 
Figure 3: Photograph of breakfast at an event hosted by PFC and a local business.

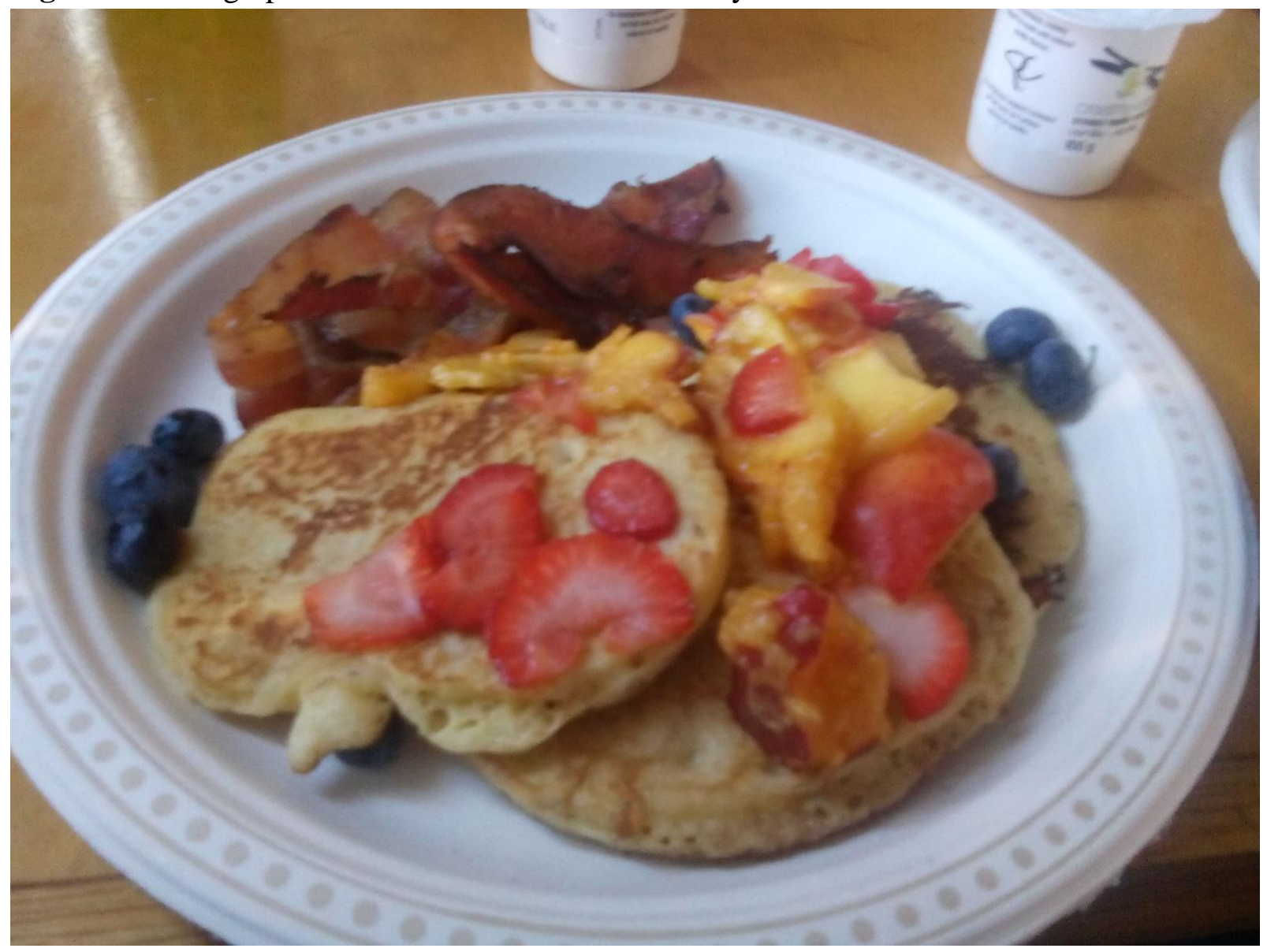

Knowledge of, and access to, other community resources was facilitated through interactions at PFC. Information about programs, resources, and services in the community were shared during interactions with other neighbours. Information and referrals were also made available through posters and pamphlets at PFC and through interactions with volunteers or staff members. Participants also discussed the need for non-food essential items, such as toothbrushes, diapers, feminine hygiene products, and toilet paper. Moreover, the importance of the additional non-food resources available at PFC, including access to computers and books in the waiting area, emerged in the data. The photograph in Figure 4 shows the availability of books and community information in the waiting area.

I just felt like a sense of community when I first came here it was just like I felt safe and sometimes I haven't been able to feel that way. So, I felt like this was my hub, like a ticket to know the whole community.

(Participant 11) 
Figure 4: Photograph of the library in the PFC waiting area.

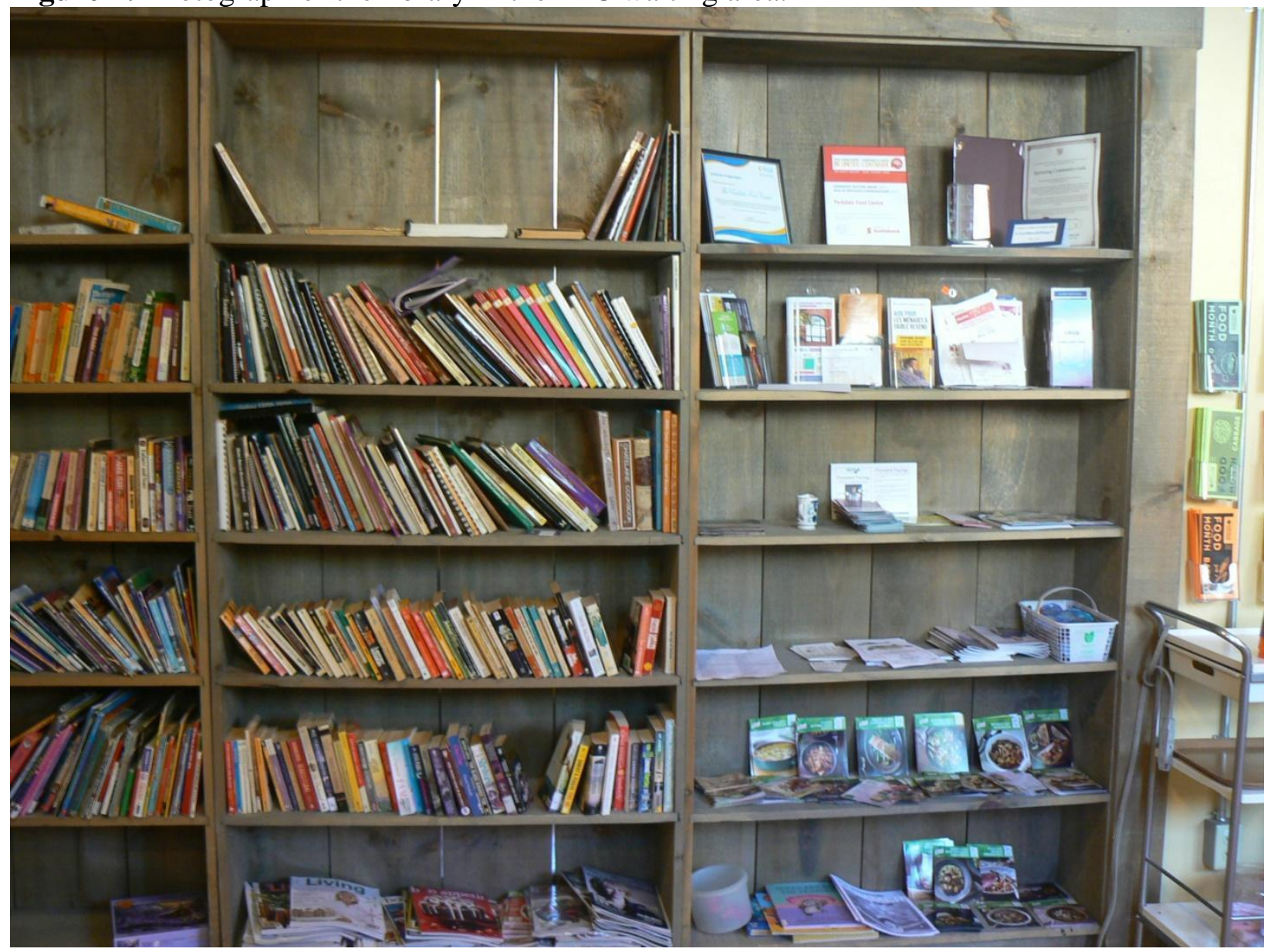

In addition to connections to other community resources or programs, seven out of 12 participants found employment, educational, or volunteering opportunities through connections made at PFC. Participants explained volunteering at PFC was encouraged among neighbours, and several explained that volunteering made them feel involved and increased their social connections in the community.

I volunteer here now every morning or in the evening. Because of the volunteering...I've made more friends. (Participant 10)

There's always extra volunteers. They're all volunteers there, everybody is kind of helping out everybody. Everybody that goes there [PFC] is comfortable enough to lend a hand when they need to. Or if something needs to get done people are always willing to help, it's nice. (Participant 5) 


\section{Dignity}

Throughout the interviews and photovoice project, the participants discussed how a sense of dignity underlined their emotional and social experiences at PFC including their overall experience of accessing the Food Centre and in their interactions with staff and volunteers. They described this in terms of how the food was distributed as well as the inclusivity and responsiveness of PFC. Four participants compared the experience of obtaining food at PFC to picking out foods at the grocery store. This includes the experience of walking around the food centre to see what foods are available, selecting the ones they would like, and having options. Participants reported preferring the choice model compared to receiving pre-packed food hampers.

I like the way we go through and pick what we want. At the other place, it was basically they made a list of what you could have and what you wanted. Somebody would go back and fill up your bags and sort of, there you go. There was always one thing that was missing. (Participant 2)

While participants explained that the choice model was preferred, one participant noted issues they perceived with the food distribution method. As illustrated in the quote below, the participant noted concerns with long line-ups and lack of consistency in the food options available for people who do not arrive early:

I come sometimes like 45 minutes early, but there's already up to 20 people ahead of me...the first crowd leaves with all of [food available at PFC] and for the last people is like not much. It's really just what's been picked through and what remains. (Participant 5)

The welcoming and inclusive environment at PFC emerged as a common theme throughout the interviews and photovoice groups and was mentioned by all 12 participants. Participants described being greeted by friendly volunteers or staff members when first entering the centre. The physical environment at PFC was described as clean, safe, family-friendly, and comfortable. Some expressed surprise when first visiting PFC as it exceeded their expectations.

It didn't look like a food bank, it had more of a cozy feeling to it. Most food banks are kind of, you know, in the basement of the church and a sort of stale environment... [PFC is] a little bit like a living room feeling. A very friendly, welcoming feeling. So that certainly struck me. They had free coffee and a lot of food banks don't do that. (Participant 4)

Staff members and volunteers were described as non-judgemental, caring, and responsive to the needs of the neighbours and community. The participants discussed how people may 
anticipate shame before their initial visit to the food centre, but after visiting they feel they can have pride and dignity at PFC. One participant described this in the quote below:

You can see that when they first get here, they're shy. But then the next time they come, their shoulders are up, they know what this place is all about. (Participant 10)

\section{Discussion}

The themes triangulated from the interview and photovoice data reveal how people perceive the impact of PFC on their food security, the social and emotional experience of accessing the Food Centre, and facets of their lives that extend beyond basic food needs. In line with past research (Kirkpatrick \& Tarasuk, 2008), the participants from the present study described the challenges of affording a balanced diet while living with a low income. In contrast with prior literature on traditional food banks, the food received at PFC was described as healthy, including fresh fruits and vegetables. The food available at food banks has previously been described as limited in quality and as mostly pre-packaged food with little nutritional value (Gany et al., 2013; Loopstra \& Tarasuk, 2012; Tarasuk et al., 2014).

People with restricted diets (e.g., due to medical conditions or allergies) may be unable to obtain suitable food or have limited options at a traditional food bank (Gany et al., 2013; Minaker et al., 2014). In contrast, the participants of the present study indicated that PFC's choice model allowed them to obtain suitable food to meet their needs, including appropriate food options for those with restricted diets. Participants explained that with the choice model of food distribution, they received foods that are more appropriate to not only dietary restrictions but also food preferences. Thus, this model of food distribution may also reduce waste, as participants explained they were more likely to eat the foods they received at the food centre if they selected it themselves. The impact of the choice model also extended into discussions of dignity and respect. Participants explained it felt more normal and dignified to make their own choices about what foods they would like from the food centre, comparing the experience to shopping at a grocery store.

Dignity emerged as a common theme throughout the interviews and photovoice project. Previous research describes the experience of embarrassment, shame, and stigma associated with seeking assistance from a food bank (Loopstra \& Tarasuk, 2012; Van der Horst et al., 2014). However, the safe and welcoming environment at PFC, including friendly volunteers and staff, opposed the expectations of shame and stigma that people seeking assistance may hold. PFC promoted respect and dignity by seeking and listening to feedback provided by people who access the food centre as well as facilitating opportunities to get involved at the food centre. Participants provided specific examples of issues that had been raised and were then addressed 
by the food bank. Furthermore, several of the participants interviewed for this study also volunteered at PFC.

In addition to providing food assistance, participants discussed how PFC worked to foster a sense of belonging and community. Their descriptions included how the use of shared spaces (e.g., a large dining table in the waiting area), and an atmosphere that is perceived as welcoming and non-judgmental, served to facilitate a friendly and social environment. For example, participants reported dropping into PFC for social reasons, outside of times when they visited to obtain food. The programs and activities facilitated by PFC further served to foster social interaction among community members. Programs that facilitate social interaction may serve to address unmet needs for some individuals, as food insecure populations often report lower levels of social support (Boston et al., 2013; Gichunge et al., 2015; Leung et al., 2015). Many participants described their challenges with lack of support, and how the spaces and programs for social interaction at PFC were a needed resource in the community.

Food banks may serve as effective access points to target populations who may benefit from a range of food, health, and social programs. While food assistance is generally what brings people to access a food bank for the first time, participants explained that they obtained more than emergency food assistance from PFC. PFC offered access to additional programming, relevant information, and connections to meet food, health, and social needs beyond their shortterm food insecurity. While food banks may not offer a solution to the problem of food insecurity, these existing agencies may be effective locations to target health and social resources for those impacted by food insecurity. These types of services may serve to address longer-term needs and, for some, cultivate a sense of empowerment that encourages them to pursue educational or employment opportunities. In turn, these pursuits may contribute to addressing the causes of food insecurity.

Participants acknowledged that the presence of the food centre within the community was an important factor in PFC having the capacity to offer the foods, products, and programs described above. In addition to creating a sense of belonging within the centre itself, the team at PFC has made efforts to connect with community groups and businesses. These connections have allowed PFC to improve the variety, quality, and quantity of food available, the availability of other products (e.g., hygiene products), and create opportunities to connect people with programs and events facilitated in collaboration with community businesses and groups. Connections within the community and broader area (e.g., municipality) may also serve to spread awareness and advocate for changes to better address issues of food insecurity. While themes addressing advocacy, efforts did not emerge in the present study, future research may examine cases of food banks or food centres that integrate advocacy into their work and the impacts of such efforts.

There are limitations that should be considered when interpreting the results of this study. First, the generalizability of these results may be limited due to the small, convenience sample that was recruited. Participants contrasted their experiences at PFC to their prior food bank experiences; however, these results were based on participant recollection alone as a comparison 
food bank was not included in the present study. Moreover, participants did not provide much description of aspects of PFC they find to be negative or in need of improvement. Data collection occurred within the same building as PFC, which may have contributed to how comfortable participants felt with being critical. This may be a potential bias that should be noted when interpreting the findings presenting above. The results from this qualitative study may inform future studies. For example, future research may consider longitudinal methods to examine different approaches amongst food banks to provide further insight into how centres, such as PFC, may impact the food security and well-being of the people who access them.

\section{Conclusions}

The present study elucidates the impact of one community food centre on the diet, health, and social experiences of the people who access the centre. Participants explained that PFC not only made a difference in their access to food, including fresh and nutritious food options, but also served an important role in their community. The food centre was described as a place for social interaction and community connections to resources. While food assistance may not provide a solution to long-term food insecurity, these findings demonstrate how an existing emergency food assistance program can expand its approach and programming to address community food insecurity and related needs.

\section{References}

Bazerghi, C., McKay, F. H., \& Dunn, M. (2016). The role of food banks in addressing food insecurity: A systematic review. Journal of Community Health, 41(4), 732-740. DOI: 10.1007/s10900-015-0147-5

Boston, P. Q., Rahman, S. M., Brown, C. P., Lopez, I. A., \& Musingo, M. (2013). The ability of women to overcome household food insecurity: Social support \& social networks. Universal Journal of Public Health, 1(4), 166-171. DOI: 10.13189/ujph.2013.010402

Campbell, E. C., Ross, M., \& Webb, K. L. (2013). Improving the nutritional quality of emergency food: A study of food bank organizational culture, capacity, and practices. Journal of Hunger \& Environmental Nutrition, 8(3), 261-280. DOI: 10.1080/19320248.2013.816991

Caspi, C. E., Davey, C., Friebur, R., \& Nanney, M. S. (2016). Results of a pilot intervention in food shelves to improve healthy eating and cooking skills among adults experiencing food insecurity. Journal of Hunger \& Environmental Nutrition, 12(1), 77-88. DOI: 10.1080/19320248.2015.1095146

Cheung, H. C., Shen, A., Oo, S., Tilahun, H., Cohen, M. J., \& Berkowitz, S. A. (2015). Food insecurity and body mass index: A longitudinal mixed methods study, Chelsea, 
Massachusetts, 2009-2013. Preventing Chronic Disease, 12, E125. DOI: $10.5888 /$ pcd12.150001

Dave, J. M., Thompson, D. I., Svendsen-Sanchez, A., McNeill, L. H., \& Jibaja-Weiss, M. (2017). Development of a nutrition education intervention for food bank clients. Health Promotion Practice, 18(2), 221-228. DOI: 10.1177/1524839916681732

Feeding America. (2014). Hunger in America 2014. https://www.feedingamerica.org/research/hunger-in-america

Food Banks Canada (2016). HungerCount 2016. https://www.foodbankscanada.ca/Hunger-inCanada/Research/HungerCount-2016.aspx

Gany, F., Bari, S., Crist, M., Moran, A., Rastogi, N., \& Leng, J. (2012). Food insecurity: Limitations of emergency food resources for our patients. Journal of Urban Health, 90(3), 552-558. DOI: 10.1007/s 11524-012-9750-2

Gichunge, C., Harris, N., Tubei, S., Somerset, S., \& Lee, P. (2015). Relationship between food insecurity, social support, and vegetable intake among resettled African refugees in Queensland, Australia. Journal of Hunger \& Environmental Nutrition, 10(3), 379-389. DOI: 10.1080/19320248.2014.929544

Greater Vancouver Food Bank (2016). Social innovation in food banks. https://www.foodbank.bc.ca/wp-content/uploads/2016/09/Social-Innovation-Scan-ofFood-Banks-V3.0-FINAL-clean.pdf

Handforth, B., Hennink, M., \& Schwartz, M. B. (2013). A qualitative study of nutrition-based initiatives at selected food banks in the Feeding America network. Journal of the Academy of Nutrition and Dietetics, 113(3), 411-415. DOI: 10.1016/j.jand.2012.11.001

Hansen-Ketchum, P., \& Myrick, F. (2008). Photo methods for qualitative research in nursing: An ontological and epistemological perspective. Nursing Philosophy, 9(3), 205-213.DOI: 10.1111/j.1466-769X.2008.00360.x

Hergenrather, K. C., Rhodes, S. D., Cowan, C. A., Bardhoshi, G., \& Pula, S. (2009). Photovoice as community-based participatory research: A qualitative review. American Journal of Health Behavior, 33(6), 686-698.DOI: 10.5993/ajhb.33.6.6

Holben, D. H. (2012). Food bank users in and around the lower mainland of British Columbia, Canada, are characterized by food insecurity and poor produce intake. Journal of Hunger \& Environmental Nutrition, 7(4), 449-458. DOI: 10.1080/19320248.2012.732925

Kirkpatrick, S. I., \& Tarasuk, V. (2008). Food insecurity is associated with nutrient inadequacies among Canadian adults and adolescents. The Journal of Nutrition, 138(3), 604-612. DOI: 10.1093/jn/138.3.604

Knoblock-Hahn, A., Murphy, A., Brown, K., \& Medrow, L. (2017). Integrative nutrition and health models targeting low-income populations: A pilot intervention in three food banks. Journal of the Academy of Nutrition and Dietetics, 117(1), 128-131. DOI: 10.1016/j.jand.2016.04.011

Laraia, B. A. (2013). Food insecurity and chronic disease. Advances in Nutrition: An International Review Journal, 4(2), 203-212.DOI: 10.3945/an.112.003277

Leung, C. W., Epel, E. S., Willett, W. C., Rimm, E. B., \& Laraia, B. A. (2015). Household food insecurity is positively associated with depression among low-income supplemental nutrition assistance program participants and income-eligible nonparticipants. The Journal of Nutrition, 145(3), 622-627. DOI: 10.3945/jn.114.199414 
Levkoe, C., \& Wakefield, S. (2011). The community food centre: Creating space for a just, sustainable, and healthy food system. Journal of Agriculture, Food Systems, and Community Development, 2(1), 249-268. DOI: 10.5304/jafscd.2011.021.012

Loopstra, R., \& Tarasuk, V. (2012). The relationship between food banks and household food insecurity among low-income Toronto families. Canadian Public Policy, 38(4), 497-514. DOI: $10.3138 / C P P .38 .4 .497$

Martin, K. S., Wu, R., Wolff, M., Colantonio, A. G., \& Grady, J. (2013). A novel food pantry program: food security, self-sufficiency, and diet-quality outcomes. American Journal of Preventive Medicine, 45(5), 569-575. DOI: 10.1016/j.amepre.2013.06.012

McIntyre, L., Tougas, D., Rondeau, K., \& Mah, C. L. (2016). "In"-sights about food banks from a critical interpretive synthesis of the academic literature. Agriculture and Human Values, 33(4), 843-859. DOI: 10.1007/s10460-015-9674-z

McKenzie, M., Tulsky, J. P., Long, H. L., Chesney, M., \& Moss, A. (1999). Tracking and follow-up of marginalized populations: a review. Journal of Health Care for the Poor and Underserved, 10(4), 409-429. DOI: 10.1353/hpu.2010.0697

Minaker, L. M., Elliott, S. J., \& Clarke, A. (2014). Exploring low-income families' financial barriers to food allergy management and treatment. Journal of Allergy, 2014(160363), 1-8. DOI: $10.1155 / 2014 / 160363$

Mukoya, M. N., McKay, F. H., \& Dunn, M. (2017). Can giving clients a choice in food selection help to meet their nutritional needs?: Investigating a novel food bank approach for asylum seekers. Journal of International Migration and Integration, 18(4), 981-991. DOI: 10.1007/s12134-017-0515-3

Neter, J. E., Dijkstra, S. C., Dekkers, A. L. M., Ocké, M. C., Visser, M., \& Brouwer, I. A. (2017). Dutch food bank recipients have poorer dietary intakes than the general and lowsocioeconomic status Dutch adult population. European Journal of Nutrition, 57(8), 27472758. DOI: 10.1007/s00394-017-1540-X

Palibroda, B, Krieg, B., Murdock, L, and Havelock, J. (2009). A practical guide to Photovoice, sharing pictures, telling stories and changing communities. Prairie Women's Health Centre of Excellence.

Parkdale Food Centre. (2019). Parkdale Food Centre. https://parkdalefoodcentre.ca/aboutus/ Pryor, L., Lioret, S., van der Waerden, J., Fombonne, É., Falissard, B., \& Melchior, M. (2016). Food insecurity and mental health problems among a community sample of young adults. Social Psychiatry and Psychiatric Epidemiology, 51(8):1073-1081. DOI: 10.1007/s00127-016-1249-9

Purdam, K., Garratt, E. A., \& Esmail, A. (2015). Hungry? Food insecurity, social stigma and embarrassment in the UK. Sociology, 50(6), 1072-1088. DOI:

$10.1177 / 0038038515594092$

Ramsey, R., Giskes, K., Turrell, G., \& Gallegos, D. (2011). Food insecurity among Australian children: Potential determinants, health and developmental consequences. Journal of Child Health Care, 15(4), 401-416. DOI: 10.1177/1367493511423854

Riches, G., \& Silvasti, T. (Eds.). (2014). First world hunger revisited: Food charity or the right to food? $2^{\text {nd }}$ ed. New York, NY: Palgrave Macmillan.

Roncarolo, F., Bisset, S., \& Potvin, L. (2016). Short-term effects of traditional and alternative community interventions to address food insecurity. PLOS ONE, 11(3), e0150250. DOI: 10.1371/journal.pone.0150250 
Roshanafshar, S., \& Hawkins, E. (2015). Food insecurity in Canada. Health at a Glance. Statistics Canada catalogue no. 82-624-X. ISSN 1925-6493. https://www.feedopportunity.com/wp-content/uploads/2016/11/Stats-Can-Food-InsecurityReport.pdf.

Seligman, H. K., Laraia, B. A., \& Kushel, M. B. (2010). Food insecurity is associated with chronic disease among low-income NHANES participants. The Journal of Nutrition, 140(2), 304-310. DOI: 10.3945/jn.109.112573

Tarasuk, V., Dachner, N., Hamelin, A.-M., Ostry, A., Williams, P., Bosckei, E., Poland, B, \& Raine, K. (2014). A survey of food bank operations in five Canadian cities. BMC Public Health, 14(1), 1-11. DOI: 10.1186/1471-2458-14-1234

Tarasuk, V., Mitchell, A., \& Dachner, N. (2016). Household food insecurity in Canada, 2014. https://proof.utoronto.ca/wp-content/uploads/2016/04/Household-Food-Insecurity-inCanada-2014.pdf

Thomas, D. R. (2006). A general inductive approach for analyzing qualitative evaluation data. American Journal of Evaluation, 27(2), 237-246. DOI: 10.1177/1098214005283748

Van der Horst, H., Pascucci, S., \& Bol., W. (2014). The "dark side" of food banks? Exploring emotional responses of food bank receivers in the Netherlands. British Food Journal, 116(9), 1506-1520. DOI: 10.1108/BFJ-02-2014-0081

Wang, C., \& Burris, M. A. (1997). Photovoice: Concept, methodology, and use for participatory needs assessment. Health Education \& Behavior, 24(3), 369-387. DOI: 10.1177/109019819702400309 